\title{
Exploring student preferences when calculating expectation values using a computational features framework
}

\author{
Benjamin P. Schermerhorn $\odot,{ }^{1,2}$ Gina Passante $\odot,{ }^{2}$ Homeyra Sadaghiani $\odot,{ }^{1}$ and Steven J. Pollock ${ }^{3}$ \\ ${ }^{1}$ Department of Physics \& Astronomy, California Polytechnic University Pomona, \\ Pomona, California 91768, USA \\ ${ }^{2}$ Department of Physics, California State University, Fullerton, Fullerton, California 92831, USA \\ ${ }^{3}$ Department of Physics, University of Colorado, Boulder, Boulder, Colorado 80309, USA
}

(Received 10 July 2019; published 26 November 2019)

\begin{abstract}
Undergraduate quantum mechanics (QM) uses a variety of notations, each with their own advantages and constraints, for representing quantum states and carrying out individual calculations. An example of this can be seen when calculating expectation values, which can be solved using several different methods. Analysis of written exam data given at three universities (teaching spins-first QM) showed students were more likely to use matrix or integral calculation in situations where it is much simpler to use a summation method. By adapting the structural features framework by Gire and Price, we are able to answer questions regarding how and why students use different methods. We analyze student responses to expectation value problems administered in interviews conducted at two universities in the middle and end of the semester to highlight specific areas of difficulty and features of the methods which led to students' choices of one method over another.
\end{abstract}

DOI: 10.1103/PhysRevPhysEducRes.15.020144

\section{INTRODUCTION}

Quantum mechanics utilizes a variety of representations. Depending on the context, quantum systems can be defined by the Dirac notation, vectors and matrices, or continuous functions. These notations are then used to carry out calculations, including normalization, finding probabilities, and describing a time evolved state. Gire and Price identified four different structural features as a way to categorize the benefits and drawbacks of these notations [1]. In this paper, we present an adaptation of this framework in order to describe how students interact with a specific task by focusing on what we call computational features. The adapted framework will be detailed in Sec. III.

The computational example used in this paper is the calculation of expectation values. Given the probabilistic nature of quantum mechanics, students are commonly asked to carry out expectation value calculations as a way to describe the physical system. Such problems provide a rich context for research, given that an expectation value can be calculated for any observable in a variety of different contexts. For example, given a state defined in terms of a spin- $1 / 2$ system, expectation values can be

Published by the American Physical Society under the terms of the Creative Commons Attribution 4.0 International license. Further distribution of this work must maintain attribution to the author(s) and the published article's title, journal citation, and DOI. calculated for a measurement of spin along any direction (e.g., $x$ axis or an axis along the $\hat{n}$ direction). Given a wave function defined for a one-dimensional infinite square well potential energy, expectation values can be calculated for position, energy, and momentum.

Depending on the context, there are several different methods that can be used to carry out the calculation. Most abstractly, the expectation value of an operator $\hat{A}$ for pure states is written in Dirac notation as

$$
\langle\hat{A}\rangle=\langle\psi|\hat{A}| \psi\rangle
$$

Three of the most common methods derived from this representation are what we refer to as the summation method, the matrix method, and the integration method. Each method has different advantages and constraints, some specific to individual contexts (spin or wave functions) and the types of observables (discrete or continuous). Additionally, each method makes use of different mathematical operators and the amount of algebraic steps required may vary between methods.

Given any discrete system, the expectation value can be evaluated as the summation of possible measurement outcomes weighted by their probabilities. This is the value one would obtain from measuring a large number of identically prepared systems and averaging the outcomes. Using the eigenequation $\hat{A}\left|a_{i}\right\rangle=\lambda_{i}\left|a_{i}\right\rangle$, where $\left|a_{i}\right\rangle$ is an eigenstate of operator $\hat{A}$ with eigenvalue $\lambda_{i}$, the expression for summation is connected to Dirac notation as follows: 


$$
\begin{aligned}
\langle\hat{A}\rangle & =\langle\psi|\hat{A}| \psi\rangle \\
& =\sum_{i} \sum_{j}\left\langle\psi \mid a_{i}\right\rangle\left\langle a_{i}|\hat{A}| a_{j}\right\rangle\left\langle a_{j} \mid \psi\right\rangle \\
& =\sum_{i} \lambda_{i}\left|\left\langle a_{i} \mid \psi\right\rangle\right|^{2}=\sum_{i} \lambda_{i} p_{i} .
\end{aligned}
$$

This method is particularly useful if you already know the eigenvalues and probabilities, or can solve for them easily.

The expectation values for discrete systems can also be found using the matrix method. For a two-state system, such as a spin- $1 / 2$ particle, the state $|\psi\rangle$ can be represented as a two-dimensional vector and the operator as a two-bytwo matrix

$$
\langle\hat{A}\rangle=\langle\psi|\hat{A}| \psi\rangle=\left(\begin{array}{ll}
a^{*} & b^{*}
\end{array}\right)\left(\begin{array}{ll}
A_{11} & A_{12} \\
A_{21} & A_{22}
\end{array}\right)\left(\begin{array}{l}
a \\
b
\end{array}\right) .
$$

This method has the advantage of not needing to know the eigenvalues and probabilities, but it does require that the system be discrete. A disadvantage to this method is that the size of the matrices scale exponentially with the dimension of the system.

When the states are written as functions to represent a continuous basis, the expectation value can be evaluated using integration, as seen in Eq. (4). While we have written the integral in terms of $x$, it could also be written in as a function of other variables, such as momentum

$$
\langle\hat{A}\rangle=\langle\psi|\hat{A}| \psi\rangle=\int \psi^{*}(x) \hat{A} \psi(x) d x .
$$

The integration method will always work if you have a function. The drawbacks are that these integrals can get complicated quickly and often require integral tables or software in order to solve.

In some situations only one method is viable. However, other times multiple methods may be used. In such cases, each method presents distinct advantages and drawbacks as outlined above, which can influence individual preference.

Calculation of an expectation value not only requires connecting the physical information given in the problem and executing the mathematical operations, but it also requires students to make decisions about which method is most appropriate. To explore the intricacies of each method, as well as the choices students make when selecting a method to solve for expectation value, we seek to answer the following questions.

1. What possible challenges do students face when implementing different methods?

2. What aspects of a given method influence students' preferences?

3. If students use a method that is not their preferred method, what influences their choice?
In Sec. II we describe the results of previous research into the frequency with which students use the different methods for computing the expectation value. We describe the computational features framework in Sec. III. This is followed by a description of the research design and methodology in Sec. IV and the results in Sec. V. We conclude with implications for instruction in Sec. VIII.

\section{PREVIOUS RESEARCH AND PRELIMINARY INVESTIGATIONS}

While most of the research on students' work with the expectation value has identified difficulties with students understanding, little work has specifically addressed students' use of or preferences for methods of calculating expectation values. In a survey administered to graduate students at the beginning of a graduate quantum mechanics course, Singh found that only $39 \%$ of students were able to calculate the expectation value of energy at a time $t$, for a given two state superposition described at $t=0$ [2]. Prior to this question, students had already been asked about the probabilities and values that could be measured for the state at $t=0$; a task which only $67 \%$ of students were reported to do correctly. The methods that students used to calculate the expectation value were not reported, beyond indicating that "many students... calculated the $\langle E\rangle$ by brute force: first writing $\langle E\rangle=$ $\int_{-\infty}^{\infty} \psi(x)^{*} \hat{H} \psi(x) d x$ " [2]. Singh reported that $17 \%$ of the students in interviews would write $\langle\psi|H| \psi\rangle$ or $\langle\psi|E| \psi\rangle$ but would then be unsure of how to proceed. In another study exploring the time dependence of expectation values, Singh reported that few students remembered that for energy eigenstates every operator has a timeindependent expectation value [3].

Other studies have found that students had difficulty distinguishing the measured value, the probability of measuring it, and the expectation value [4,5]. Singh further reported the results of one question where students were asked to qualitatively describe possible position values and the probability of measuring them. $11 \%$ of students tried to find the expectation value in place of the probability [6]. Various research has also shown that students do not know or apply the definition of the expectation value as the weighted average over many measurements $[5,7,8]$. Sadaghiani and Bao found that students also confuse the terms of "expectation value," "probability density," and "uncertainty" [9].

Work by Gire and Price [1], carried out in the context of an energy expectation value problem in the infinite square well potential energy, found that students generally needed prompting to employ matrix notation in this context and sometimes mixed up the order of the vectors and the operators. They also noted that students would often write the Dirac expression $\langle\psi|\hat{A}| \psi\rangle$ as a template for matrix or wave function notation. In other cases, students have been 
shown to have difficulty using and connecting the different notations [10,11].

In preliminary investigations across three institutions we have studied the prevalence of the methods students use when answering a variety of expectation value questions on exams [12]. We analyzed student responses to 13 different exam questions in contexts ranging from spin- $1 / 2$ particles to the infinite square well potential energy and an electron bound in a hydrogen atom. For questions in the context of position-space wave functions, we found that if asked for the expectation value of energy when the state was written in the energy eigenbasis, there was a clear preference for using the summation method as $75 \%(98 / 129)$ of the students used this method. A very small number of students (5\%) attempted to answer these questions with integration and they were ultimately unsuccessful. When the question asked for the expectation value of position, almost all students (90\%) used integration to solve the problem.

For questions in the context of spin-1/2 particles, the matrix multiplication method and the summation method were both popular choices, however on almost all questions, matrices were more common. For example, in a question where the state was written in the eigenbasis of the operator (in this case, $S_{z}$ ), approximately $50 \%$ of the students from one institution used matrix multiplication to solve for the expectation value and $85 \%$ of the students at another institution used matrix multiplication on the same question. In another case where students were asked to find $\left\langle S_{y}\right\rangle$ for a state given in the $z$ basis after being asked to find all relevant probabilities, we found that $55 \%$ of students used matrix notation and $32 \%$ used summation (with $10 \%$ using both methods). This was similar across two administrations of the question. Furthermore, there were just as many errors in student work when they used summation method as when they used matrix method. In the few cases where students used both methods, they would often label the summation method as a "check" to their work.

Our analysis suggests that students' choices are dependent on the question wording, notation, and information given. For example, it seemed to matter whether the state was provided in the basis of the operator of interest and what notation was used in the question prompt. All three courses were taught using a spins-first approach with similar materials and levels of interactive engagement, but there was some variation in emphasis of different methods used between the different institutions. Despite the different results across different learning environments, many students used the matrix calculation when the summation method would have been more efficient, suggesting that students may not have a deep understanding of multiple methods or be able to distinguish when one method might be simpler. Building on this work, this paper explores students' responses to interview prompts, described in Sec. IV, as a means to probe what leads students to use different methods, what challenges arise in each method, and what feeds into student preferences for different methods.

\section{THE COMPUTATIONAL FEATURES FRAMEWORK}

To better analyze how students implement and understand the various methods for calculating expectation values, we adapt a framework from Gire and Price that identifies the structural features of the different notations used in quantum mechanics [1]. In this section we outline the original framework for addressing the structural features, as well as the manner in which we adapted it to create the computational features framework. We further compare our adapted framework to other models describing the interaction between mathematics and physics to situate it in the literature.

Gire and Price identify four structural features of interest when focusing on student's work with quantum notations: individuation, externalization, compactness, and symbolic support. Dirac, wave function, and matrix notation were then labeled along these dimensions on a scale from low to high. While developed in the context of expectation value, this has been used by other researchers to explore students' use of different notations in other contexts [11].

The first structural feature, individuation, refers to the extent each of the important features of the notation are distinct, identifiable elements. Dirac notation is labeled with a high individuation because kets provide a clear representation of the states, while wave functions are labeled as low since components, such as coefficients and normalization constants, can be combined algebraically and then be difficult to distinguish [1]. The second feature, externalization, originally refers to the explicitness of the elements and features of a notation. Matrix notation is labeled low as there is typically nothing to indicate the basis for a given vector or matrix. Conversely, wave functions are labeled with a high level of externalization because the functional forms are clear. As a structural feature of the notation, compactness refers to the amount of space that is needed for writing out elements of the notation. Lastly, symbolic support was determined by how easily the notation assists students in carrying out calculation. Dirac notation is labeled as high because the bra-ket notation distinguishes inner and outer products and cues the necessary mathematical operation, while wave function notation is low given that there is no cuing or guiding feature of the notation.

The structural features framework was a natural starting point for our work given the near alignment between notations of quantum mechanics and methods for calculating the expectation value (e.g., wave function notation and integration). However, alignment between the notation and the method is not universal, as the summation method has no counterpart. Further, in reviewing the data from our interviews we noticed that there were different factors that 
affected computation and that students' interactions with computation were not strictly comparable to their interactions with notation. The goal of our framework is to take these factors into consideration.

In adapting the framework from describing the abstracted notation to describing students use of different methods for computation, the focus is transferred from the abstract mathematical features to how each student interacts with a given method. As such, we forgo the use of the highlow scale for the majority of the computational features to instead highlight what students do while implementing a given method. Table I summarizes the adapted framework and the questions used for analyzing student work. A specific application of our framework is described in Sec. IV B. This framework was developed during preliminary data analysis. It was informed by both Gire and Price's structural features framework and common responses from student interviews.

Since individuation refers to the distinctiveness of notation elements, we consider identification as an adaptation to refer to whether the elements of each equation are identifiable for students. As such, if a student can identify the variables for the probability or the eigenvalue in the summation equation, or recognize they need a row vector, matrix, and column vector in order from left to right in the matrix method, the student is successful with identification. Identification in this sense is independent of the context of the problem and solely attributed to an understanding of the components of the method.

In our modified framework, externalization is where the chosen method is contextualized for a given problem. Proper externalization aligns with correctly expressing the previously identified components appropriately. In line with Gire and Price [1], this means writing the vectors and matrices correctly and in the same basis when calculating an expectation value using matrix notation. For integration or summation, this manifests as incorporating the correct form of operators and wave functions or probabilities and values, respectively. As such, if a student mistakenly uses the probability of measuring spin up in the $z$ direction when calculating the expectation value of the $S_{y}$ operator, then we label this as being an issue with the externalization of the equation.

With the goal of assessing students' preferences for a given method of solution, we address the computation as a whole, referring to the computational compactness as a component of individual student work rather than from an expert perspective. Just as this is a factor in representations of notations, compactness is relevant when choosing a computational method. Objectively, the matrix method will typically require more space for calculation than summation notation. However, it is possible for an expert physicist or proficient student to limit matrix notation to a single line of work. Comparatively, a student could write out each step of the calculation in great detail. The ways in which computational compactness may impact students' choice of method may vary, as some students might value shorter (or more compact) calculations, while others might value the redundancy or methodical nature of a more expansive expression. In either case, this category will allow us to document both the compactness of a student's solution and reflect on whether or not they value that compactness.

The concept of symbolic support, as part of the structural features framework, is less meaningful for a computation, as a written equation provides clear mathematical direction for computation. Elements of the original symbolic support feature are accounted for in our features of identification and externalization. After a student identifies the necessary components of an equation and contextualizes them, the next step is to carry out the mathematics.

Given the goal of this work to assess students' computation and implementation of a given method, we introduce a fourth dimension of computational confidence, to describe students' comfort level with summations, matrices, or integration. This dimension was informed by a large number of students who discussed their varying level of comfort with different mathematics. Computational confidence is independent of a student's comfort with calculating the expectation value as a whole, and is instead an assessment of how comfortable the student is with the general mathematical operations, such as integration or matrix multiplication. This further allows us to draw focus

TABLE I. Definitions and targeted questions of the computational features used to analyze students' calculation of expectation value.

\begin{tabular}{ccccc}
\hline \hline & \multicolumn{1}{c}{ Identification } & Externalization & Computational confidence & $\begin{array}{c}\text { Computational } \\
\text { compactness }\end{array}$ \\
\hline $\begin{array}{c}\text { Abbreviated } \\
\text { definition }\end{array}$ & $\begin{array}{c}\text { Extent each of the elements } \\
\text { (of the expectation value } \\
\text { expression) are } \\
\text { identifiable }\end{array}$ & $\begin{array}{c}\text { Expression of elements and } \\
\text { features of the solution method } \\
\text { for the physical situation }\end{array}$ & $\begin{array}{c}\text { A measure of comfort with } \\
\text { a particular calculation }\end{array}$ & $\begin{array}{c}\text { Space taken for } \\
\text { writing and/or } \\
\text { calculation }\end{array}$ \\
$\begin{array}{c}\text { Targeted } \\
\text { question }\end{array}$ & $\begin{array}{c}\text { Do students know the } \\
\text { equation and what the } \\
\text { terms are in the equation? }\end{array}$ & $\begin{array}{c}\text { Do students appropriately } \\
\text { express the terms in a } \\
\text { given context? }\end{array}$ & $\begin{array}{c}\text { Are students comfortable } \\
\text { and/or confident with } \\
\text { the mathematics needed } \\
\text { for computation? }\end{array}$ & $\begin{array}{c}\text { How compact is the } \\
\text { space needed for } \\
\text { computation? }\end{array}$ \\
\hline \hline
\end{tabular}


to students' preferences for a given method. For example, a student who is less comfortable with carrying out matrix multiplication may prefer to calculate an integral when dealing with a superposition of two energy eigenfunctions. Another student may find matrix multiplication rather straightforward, which will affect the decisions they make regarding which method to use.

While used in the context of characterizing students' preferences, we posit that this framework is not limited to a comparison of different solution pathways, as it could be used by researchers as a way to characterize a student's implementation of a single method of solution. Features such as identification and externalization relate to students' expression of physics conceptual information within a mathematical language. However, because the computational features framework is concerned with preference, we incorporate both compactness and computational confidence to account for students' thoughts about the mathematical processes. As such, there is more attention to possible metacognition and decision making beyond listing student difficulties with computation.

Many researchers have already developed models for describing how students make connections between mathematics and physics [13-15]. While these models share similarities with each other (e.g., all account for mathematical modeling of physics concepts and pure mathematical calculation), the ACER framework [13] was designed to specifically analyze students' problem solving by breaking it into four stages: (1) Activation of the tool (referring to the equation or method of solution), (2) construction of the model, (3) execution of mathematics, and (4) reflection on results. We see a direct connection between our identification and externalization features and the "activation of the tool" and "construction of the model" phases of the ACER framework. To address our research goals, we also need to consider what leads students to "activate" a specific method of solution when there are multiple to choose from. Compactness and confidence address the "execution of mathematics" phases but focus on students' perspectives on the required mathematics. Thus, these features as a whole address students' use of a given method, from whether a student can contextualize a problem to their confidence with the mathematics involved.

\section{RESEARCH DESIGN AND METHODOLOGY}

In order to investigate what leads students to choose a particular method of solution when calculating the expectation value of an operator for a given state, interviews were conducted at two different institutions. Both institutions are large, public, Hispanic-serving, primarily undergraduate institutions. Data were collected at two points in the semester from a first upper-division quantum mechanics course. Courses at both institutions teach a spins-first approach using the Quantum Mechanics textbook by McIntyre [16]. The instructors used similar instructional materials including tutorials and multiple-choice concept test questions throughout lectures. During the semester in which interviews were collected, the expectation value was introduced in the context of spins using both the summation and matrix method at approximately the same point in the course. The integration method was introduced later in the semester once wave functions and the infinite square well had been introduced.

Individual student interviews were performed with student volunteers and consisted of two different protocols to include both the spins and wave function contexts. The first set of interviews $(N=20)$ asked questions in a spins context and were administered during weeks six through eight (of a 15 week semester), after the relevant material was covered in the courses. The second set of interviews $(N=12)$ were conducted during the last three weeks of the semester and were given in the context of position wave functions for an infinite square well. Interviews lasted between $30 \mathrm{~min}$ and $1 \mathrm{~h}$ depending on a student's proficiency with the problem. Ten students were interviewed at both points in the semester, meaning the latter set of interviews is majorly composed of the same students from the first set of interviews.

\section{A. Interview design}

For the first set of interviews students were given the following state:

$$
|\psi\rangle=\frac{3}{\sqrt{34}}|+\rangle_{y}-\frac{5}{\sqrt{34}}|-\rangle_{y} .
$$

While standard problems are typically given initially in the $z$ basis, this state was given in the $y$ basis to assess students' attention to the basis of the state when solving for the expectation value. Students were provided with an equation sheet including the matrix forms of the spin operators, $S_{x}, S_{y}$, and $S_{z}$, as well as the Dirac and matrix representations of their eigenstates, all written in the standard $z$ basis representation.

Students were first asked to describe the meaning of the symbols and numbers of the given state. They were then asked to solve for the expectation value of the $S_{y}$ operator where they could freely invoke one or more of the different methods. An efficient way to solve for $\left\langle S_{y}\right\rangle$ is to square the coefficients (to find probabilities) and use the summation method

$$
\left\langle S_{y}\right\rangle=\Sigma_{i} \lambda_{i} p_{i}=\left(\frac{\hbar}{2}\right)\left(\frac{\hbar}{2}\right)+\left(-\frac{\hbar}{2}\right)\left(\frac{25}{34}\right)=-\frac{16}{34} \frac{\hbar}{2} .
$$

The notation $\langle\hat{A}\rangle$ was not used directly in the question and only provided to three students who could not find a place to start.

Upon implementing any one method, students were asked to explain the steps they took during their calculation 
and to make sense of their answer given the initial state. Students were then asked if there were other methods by which they could have calculated the expectation value and to explain their work during the calculation. In the cases where a student invoked multiple methods of solution, they were then asked to elaborate on which method they preferred. Following this, students were asked to calculate the expectation value of $S_{z}$, using the same interview protocol. In this case, both the matrix method and the summation method require students to convert the state to the $z$ basis and lead to an answer of $\left\langle S_{z}\right\rangle=-\frac{60}{68} \frac{\hbar}{2}$.

The second set of interviews were conducted later in the semester using wave functions for an infinite square well potential energy. The protocol included two states in the context of an infinite square well of width $L$ and potential $V(x)=0$ inside the well. The first state was written as a superposition of energy eigenstates and the second was written as a standard parabolic function as follows:

$$
\begin{aligned}
& \psi_{1}(x)=\frac{3}{5} \quad \phi_{2}(x)-\frac{4}{5} \quad \phi_{3}(x), \\
& \psi_{2}(x)=\sqrt{\frac{30}{L^{5}}} \quad x(L-x) .
\end{aligned}
$$

Students were asked to find the expectation values of the energy and position operators. For this set of interviews students were given an equation sheet that included the position representation of the Hamiltonian operator in one dimension,

$$
\hat{H}=-\frac{\hbar}{2 m} \frac{\partial}{\partial x}+V(\hat{x})
$$

and the expressions for the $n$th energy eigenstate and eigenvalue for an infinite square well potential energy. Students were not asked to carry out integration once they had set up an integral expression as this was not the focus of this investigation. Students implementing summation or matrix notation for $\psi_{1}(x)$ were allowed to carry out the calculation completely, arriving at an answer of 7.2E $=$ $7.2 \frac{\pi^{2} \hbar^{2}}{2 m L^{2}}$ for a well of length $L$.

As with the interviews in the spins context, students were first asked if there were other methods they could have used and then asked about specific preferences. To see if students might be able to connect the different methods for calculating the expectation value, they were explicitly asked if matrices or summation were viable methods of solution for $\langle x\rangle$ once they had listed and applied any methods they felt were applicable. We had given $\psi_{2}(x)$ strictly in terms of position in order to see if students would attempt multiple methods when there are not easily identifiable energy eigenstates. As with $\langle x\rangle$, if students did not identify summation as a viable method for $\langle H\rangle$, they were directly asked if it was a possible solution method.

\section{B. Method of analysis}

Student interviews were videotaped and transcribed for analysis using the computational features framework described in Sec. III. Given the similarity between the courses and student populations, student data are considered collectively. Once the role of each feature was agreed upon following discussion among the authors, identification and externalization were coded as either complete, incomplete, or not applicable. Computational confidence was marked as either present or absent, and computational compactness was graded on a scale from low to high as discussed above.

Table II shows the simplified definitions of each dimension, the target question for each, and an example of data analysis for a student, Raife, invoking both matrix and summation method for a given problem. The table indicates that he first used matrix method, and then later attempted to use summation. The filled circles represent that students are aligned with the specification of the target question. In the example, identification is marked as complete for the matrix method which indicates that Raife completely defined the elements in the matrix expressions. The remaining filled circles for matrix notation identify that Raife was able to appropriately express the state as a vector and felt confident with his matrix multiplication. In line with Gire and Price, who used the context of expectation values for the infinite square well, matrix notation for a two-state system is generally of medium compactness. This provides a baseline for our analysis of students work. The "low" label in computational compactness indicates that the student carried out the standard matrix calculation but also needed extra computational space to convert the state

TABLE II. Example of data analysis for Raife's calculation of $\left\langle S_{y}\right\rangle$.

\begin{tabular}{lcccc}
\hline \hline & Identification & Externalization & Computational confidence & $\begin{array}{c}\text { Computational } \\
\text { compactness }\end{array}$ \\
\hline $\begin{array}{l}\text { Targeted } \\
\text { question }\end{array}$ & $\begin{array}{c}\text { Do students know the } \\
\text { equation and what the } \\
\text { terms are in the equation? }\end{array}$ & $\begin{array}{c}\text { Do students appropriately } \\
\text { express the terms in a } \\
\text { given context? }\end{array}$ & $\begin{array}{c}\text { Are students comfortable and/or } \\
\text { confident with the mathematics } \\
\text { needed for computation? }\end{array}$ & $\begin{array}{c}\text { How compact is the } \\
\text { space needed for } \\
\text { computation? }\end{array}$ \\
(1) Matrix & - & $\bullet$ & & Low \\
(2) Sum & $\circ$ & 0 & $\circ$ & \\
\hline \hline
\end{tabular}


to a different basis. When implementing the summation method, Raife did not include the eigenvalue as an element in his equation, and this is marked incomplete for identification. Externalization is marked with an incomplete circle for summation, since the probabilities he did use were for measurements along the $z$ axis and not the $y$ axis. Computational compactness is unmarked for the summation method to express that the student did not carry out the remaining calculation.

All students were analyzed in a similar manner for both interview protocols. We then went on to address students' preferences for a given method by attending to the reasons a student identified a method of solution as preferred and compared this to each of the four dimensions for a given method.

To address the two distinct contexts presented in interviews, we discuss students' solution of expectation value in the context of spins and in the context of wave functions independently. In each subsection, we first address the implementation of the different methods by attending to the identification, externalization, computation comfort, and compactness of the appropriate methods. In doing this we articulate the challenges and successes of students along these dimensions. Then we address students' preferences by attending to students who applied multiple methods. We further compare students' stated preferences to the completeness of their responses along the four computational features for both methods.

\section{RESULTS IN THE SPINS CONTEXT}

When solving for both $\left\langle S_{y}\right\rangle$ and $\left\langle S_{z}\right\rangle$ for the state $|\psi\rangle=\frac{3}{\sqrt{34}}|+\rangle_{y}-\frac{5}{\sqrt{34}}|-\rangle_{y}$, both the summation and the matrix method of calculation were commonly invoked by students. While not every student implemented both methods, all but one student employed at least one of the two methods and was able to make headway on the calculation. Students tended to use the same method (or methods) for both questions without discussing or considering what might be the most efficient method for the given task.

\section{A. Analysis of the matrix method for spins}

The matrix method was invoked by the majority of students interviewed in the spins context. Of the students who used both matrix and summation methods, two-thirds of them started with matrices. In total, 17 of the 20 students interviewed at the end of the spins portion of the semester used the matrix method to solve for an expectation value of both $\left\langle S_{y}\right\rangle$ and $\left\langle S_{z}\right\rangle$.

\section{Identification}

In terms of applying the matrix method, identification accounts for recognizing the elements of the solution as a successive product of a row vector, matrix, and column vector. The majority of students employing the matrix method were able to address the elements as such [Fig. 1(a)]. A few students made a minor error by identifying $\langle\psi|$ as a column vector, but immediately noticed their mistakes when attempting to carry out the mathematics. Most students spontaneously connected the order of their matrix and vectors to the Dirac form of the expectation value calculation, $\left\langle\psi\left|S_{y}\right| \psi\right\rangle$. By the time the expectation value is introduced, students have been working extensively with both Dirac and matrix representations, so it is not surprising that the majority of students are able to transition between them.

Three students, Harper, Piper, and Keith, were marked with an incomplete identification as they did not identify $|\psi\rangle$ as a vector. Both Harper and Piper treated the state as a matrix, writing the coefficients as the diagonal elements and placing zeros as the off-diagonal elements (Fig. 1(b)). Keith represented $|\psi\rangle$ as a product of a row and column vector, where $|+\rangle_{y}$ and $|-\rangle_{y}$ served as the row and column, respectively. Only Piper was able to eventually recognize and correct her mistake when the multiplication of the three matrices for $\left\langle\psi\left|S_{y}\right| \psi\right\rangle$ yielded a matrix instead of a single number as she expected for the expectation value.

Harper and Keith were two of three students who struggled to start solving for expectation value and were explicitly prompted with the notation " $\left\langle S_{y}\right\rangle$." At this point all three of the students employed the matrix method from the expanded bra-ket representation $\left\langle\psi\left|S_{y}\right| \psi\right\rangle$, suggesting that the bracket notation in $\left\langle S_{y}\right\rangle$ and the Dirac states in the expanded form are strong cues to students' use of matrix notation.

\section{Externalization}

After identifying the components of the expression, students would then need to externalize the matrix method. Externalization relates to expressing the vectors and matrices in the given context and using the appropriate basis. For the expectation value of $S_{y}$, students had to choose to use either the $z$ basis or $y$ basis for their matrices. The matrix, as provided to the students, was written in the $z$ basis, which is (a)

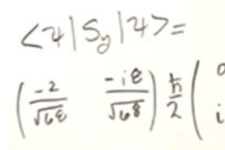

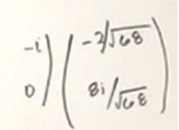

(b)

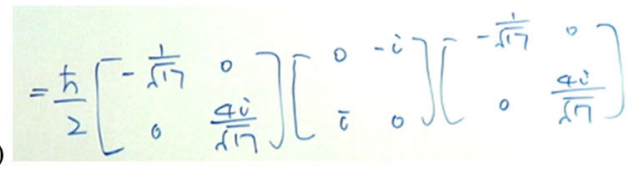

FIG. 1. Students' identification of matrix notation where $\psi$ is expressed (a) correctly as a vector and (b) incorrectly as a matrix. 
the most common form of representation when working with spin systems [16]. Using this representation requires students to convert the state $|\psi\rangle$ from the given $y$ basis to the $z$ basis. Alternatively, it is possible to work in the $y$ basis, which does not require students to convert the state, but does require students to identify the matrix representation for $S_{y}$. When calculating the expectation value of $S_{y}$, seven students worked completely in the $z$ basis and two students worked in the $y$ basis. The two students who worked in the $y$ basis did eventually convert the state to the $z$ basis when solving for $\left\langle S_{z}\right\rangle$.

Five students incorrectly used a mixed basis representation by writing $|\psi\rangle$ in the $y$ basis but the $S_{y}$ matrix in the $z$ basis, which results in an expectation value of zero. Three of these students, all who later invoked the summation method, stated that the resulting answer did not make sense since the given state suggested a higher probability of measuring the spin down state. Two were then able to correctly diagnose that the issue was due to the mismatch of basis and ultimately arrived at the correct answers.

Two of the students who mixed bases for $\left\langle S_{y}\right\rangle$ later stated the vector did need to be converted when they were solving the expectation value of $S_{z}$. When Julia was asked why she used a "different-looking" $|\psi\rangle$ for the $S_{z}$ calculation she responded that the original state "was given in the $y$ basis, but in order to find the expectation value for the $z$ basis we need the state to be in the $z$ basis because you can't operate the $z$ operator on the $y$ basis." Hence, Julia felt she could use $S_{y}$ with a state written in the $y$ basis. This suggests that some students may have a hard time differentiating the basis that a matrix is written in from the spin operator the matrix represents.

The larger number of other students mixing of the $z$ and $y$ bases when applying matrices suggests some students do not recognize or think about which basis the spin operators are written in or the need to represent vectors and matrices in the same basis for a given calculation. Furthermore, these interviews show that some students do not necessarily recognize the resulting inconsistencies from issues with basis or are unsure how to resolve them.

\section{Computational confidence}

The majority of students expressed or displayed confidence with the matrix method, stating that matrix notation and/or multiplication was familiar. Student confidence with matrices at this point in the curriculum is not surprising given the emphasis on matrices throughout the beginning of spins-first quantum instruction. This stated confidence, or lack thereof, played a large role in influencing students' preferences.

Raife: I feel more confident with this way [matrix method] because I've been doing that.

This statement was echoed by a number of students. Other students made comparisons between matrix and
Dirac notation, stating they found matrices to be a more streamlined or organized notation. Two students went as far as saying they just enjoyed the mathematics associated with matrices, as exemplified by the following quote.

Owen: I think I favor matrix multiplication because I really enjoyed linear algebra, so whenever we get to use matrices I jump on it.

Other students responded that they found matrices hard or confusing. These students were also those that struggled with representing the expression (e.g., using matrices in place of vectors), like Harper.

Harper: I understand matrices and how to multiply them and stuff but I don't know, for some reason it [matrix notation] is more confusing to me.

While Harper stated that they knew how to multiply matrices, they expressed a general lack of confidence with the use of matrices in general. Three other students shared similar lack of confidence, one of whom emphasized that she would purposefully use matrix more because she was often troubled by it.

Computational confidence divided students along the lines of whether or not they were confident in carrying out linear algebra, however this did not always mean they were proficient with the matrix method. The three students who arrived at incorrect answers due to mixing basis representation and did not invoke summation, expressed high levels of confidence in their understanding of matrix calculation.

\section{Compactness}

In terms of compactness, matrices in general need a fair amount of space for computation. Even more space was required in order to convert the vector to match the basis of the matrix, which most students represented in the $z$ basis. While two students were able to perform a very compact calculation of $\left\langle S_{y}\right\rangle$ by writing their vectors and operator in the $y$ basis (Fig. 2), this was not common and these students eventually performed a basis conversion when calculating $\left\langle S_{z}\right\rangle$.

We defined a medium level of compactness as carrying out matrix method when the states and matrix are already in the same basis. This is consistent with Gire and Price's labeling of matrix notation as medium compactness for a two state system [1]. Including the need for a basis conversion, the subsequent expectation value calculation was labeled with a low compactness to account for the extra space the conversion requires. In some cases compactness

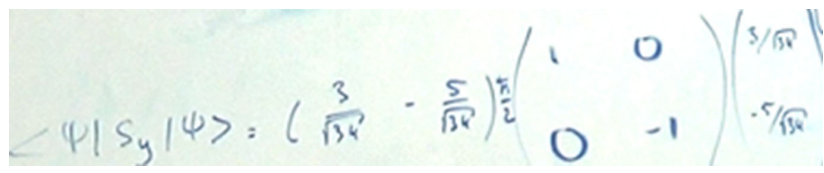

FIG. 2. Mark's expectation value calculation where he expressed everything in the $y$-basis representation. 
was labeled "very low" when students would convert both $|\psi\rangle$ and $\langle\psi|$ in Dirac notation rather than convert the state once and use then write the complex conjugate.

The increased space needed to carry out a calculation using the matrix method is why most experts would consider the matrix method a less efficient calculation. For the two students converting $S_{y}$, even with the extra space involved, the time it took to carry out the matrix calculation was minimal. Both students immediately recognized they could represent the $S_{y}$ matrix in its own basis and completed the calculation in fewer than $3 \mathrm{~min}$. This experience was limited to these two students as most others, even those with high confidence, would spend much longer on converting the state and carrying out the calculation.

\section{B. Analysis of the summation method for spins}

The summation method was utilized less often by students $(N=12)$, in line with previous exam data described in Sec. II. As with the matrix method, students not arriving at the correct answer had various difficulties with the identification and externalization aspects of the summation method, typically related to identifying and expressing the eigenvalues during calculation. Additionally, computational confidence was more mixed among students depending primarily on the amount of practice students felt they had with the summation method.

\section{Identification}

Proper identification for the summation method required that students be able to name the terms $p_{i}$ and $\lambda_{i}$ as the probabilities and the eigenvalues for a given state and operator [Eq. (2)]. Most students, upon indicating that summation was a possible method, were able to articulate that the components of the method were probabilities and eigenvalues. Only one student, Raife, did not identify that the equation for the summation method needed both eigenvalues and probabilities. He initially included the eigenvalues in the calculation but when asked to explain his work, argued that they were not part of his final answer.

Interviewer: So why is that $[\hbar / 2]$ in there?

Raife: Um, it [the $+\hbar / 2]$ just tells me this is the positive [state's probability]. It doesn't need to be there. Just for me to know that this is positive [state's probability Fig. 3]

Raife pointed to the probabilities, suggesting that the eigenvalues were only written in his equation to tell him which probability to make positive and which to make negative. We label Raife's struggle as an issue with identification because Raife was not able to recognize the components of summation. Almost all of the remaining students implementing the summation method wrote out the equation before implementation and were able to identify the variables and sometimes even wrote out "probabilities" and "values" in place of $p_{i}$ and $\lambda_{i}$.

\section{Externalization}

After identifying the components of the summation equation, students need to represent them for the given operator and state. The eigenvalues for both expectation values of the spin in the $y$ and $z$ directions are $+\hbar / 2$ and $-\hbar / 2$. To find the probabilities for the $\left\langle S_{y}\right\rangle$ calculation, students can simply square the given coefficients in the state $|\psi\rangle$. The majority of the students used this method for $\left\langle S_{z}\right\rangle$ as well, since they had already converted the state to the $z$ basis.

Five students struggled with the externalization of the expectation value using summation notation. Only two of these students were identified with externalization issues related to expressing the probabilities for the summation method. Raife, mentioned above, incorrectly used the probabilities for spin in the $z$ direction when calculating $\left\langle S_{y}\right\rangle$. The second student Tristan began the calculation for $\left\langle S_{z}\right\rangle$ with the assumption that the probabilities for the state when measured along the $z$ axis were split 50/50. This is an idea that is common among students early in the course when they are first learning about the Stern-Gerlach apparatus.

Three other students, Piper, Duncan, and Calleigh, each identified $\lambda_{n}$ as the eigenvalue in the equation for summation but struggled with determining what the eigenvalues were for this problem. Summation was the first method that Piper attempted, but she was unsure about what value to use as the eigenvalue, although she did suggest that there should be a positive and a negative value. This ultimately led her to use the matrix method. Duncan, being uncomfortable with the summation, used $\hbar / 2$ as the sole eigenvalue for both the $S_{y}$ and $S_{z}$ operators. Rather than carrying out the calculation, Duncan then set the summation equal to the result from his incorrect matrix calculation saying the result would be the same. Calleigh's matrix multiplied the operator $S_{y}$ by $|\psi\rangle$ and used the resulting vector elements as her eigenvalues (Fig. 4).

Mislabeling of other quantities as eigenvalues was prevalent in other parts of the interview as well. As part of the protocol, in the beginning of the interview students were asked to describe the components of the state. Five
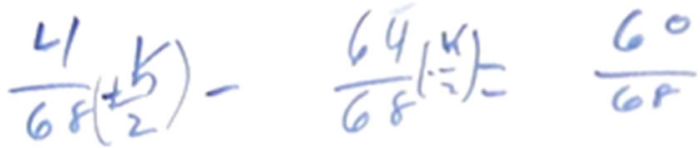

FIG. 3. Raife's use of the summation method for $\left\langle S_{y}\right\rangle$ where he uses probabilities for spin in the $z$ direction. He includes the eigenvalues to label probabilities but states they do not belong in the calculation. 


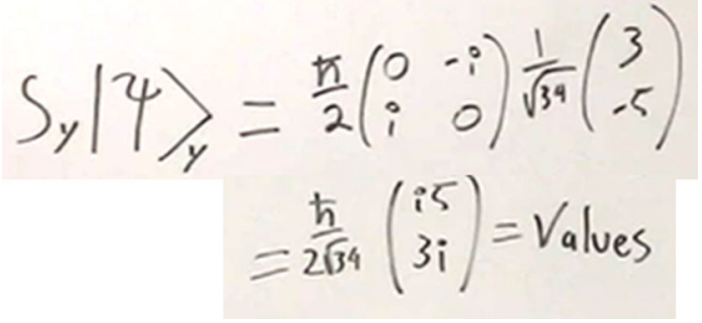

FIG. 4. Calleigh's attempt at the summation method where she identifies that values and probabilities are needed but expressed the values as the results of a calculation.

students, who did not use the summation method (and are therefore not described further in this section), referred to the coefficients in the state, $|\psi\rangle=\frac{3}{\sqrt{34}}|+\rangle_{y}-\frac{5}{\sqrt{34}}|-\rangle_{y}$, as the eigenvalues. This further suggests a more general struggle with identifying eigenvalues. It is likely that if these students had attempted to use the summation method, they would have struggled with externalization.

Some students do not recognize when the wrong values or probabilities have been included. However, if students are aware that they do not know how to represent the eigenvalues or probabilities, it is much harder for them to carry out the calculation. In contrast, externalization issues in the matrix method can be less obvious to students, since, as stated by Gire and Price, basis is a hidden element of matrix notation. This results in more students arriving at an incorrect answer, but being confident in the result, when using the matrix multiplication method.

\section{Computational confidence}

As with the matrix method, students were split into two groups related to computational confidence. They were either very confident in their application of summation since they found the associated mathematics easy, or were unsure of how to apply the summation method because of a stated lack of practice with implementing it. Four students expressed a lack of confidence in the application of summation notation.

Leanne: I'm not as comfortable using that formula [summation] just because it is not something I've practiced nearly as much. Like this approach [matrix method] is like, we've done this several, several times, but the other approach I'm not as familiar with.

Interviewer: Is there a part of it makes you less comfortable with it [summation]?

Leanne: Just lack of familiarity really. It's something like, it feels like it showed up in the textbook and it's showed up couple times in lecture but...I can't think of any times where we've used that formula specifically...I'm not sure how to use it when I need to.

Despite arriving at correct answers that were consistent with the results of their matrix notation, both Tristan and
Leanne admitted they more often used matrix notation and did not feel confident with summation notation. They were even hesitant to apply it for the subsequent calculation of $\left\langle S_{z}\right\rangle$ due to a lack of familiarity with the method. Most of the students expressing a lack of confidence with summation were those who struggled with the externalization of the method.

Other students using the summation method were confident with its invocation and the simpler mathematics involved. For Mark, this confidence extended to a comfort with the method as a whole because it felt more intuitive to him.

Mark: I like the sum, because I think it is easier and has a more physical interpretation with heights [referring to bar graph he'd drawn] and probability and then whenever I'm doing calculations and stuff it's easier for me to do.

Mark was able to connect the mathematics of the calculation to a bar graph representation and the physical interpretation of the expectation value as the weighted average over many measurements. Students who felt more confident found the simplified mathematics of the summation method to be easier and were often easily able to connect the result to the physical interpretation.

\section{Compactness}

Calculating $\left\langle S_{y}\right\rangle$ using the summation method was a highly compact process given that it only necessitated squaring the coefficients in front of $|+\rangle_{y}$ and $|-\rangle_{y}$ to find the probabilities. For the calculation of $\left\langle S_{z}\right\rangle$, students needed to solve for the probabilities, sometimes by writing the state in the $z$ basis. One common method involved substituting the $z$ basis representation of the $|+\rangle_{y}$ and $|-\rangle_{y}$ states into the given state in order to arrive at the appropriate $z$ basis representation. Students then squared the coefficients to find probabilities. Alternatively, a few students bypassed the conversion of the state by calculating $|\langle \pm \mid \psi\rangle|^{2}$ to find the probabilities directly.

We labeled the conversion or projection onto the $z$ basis states as decreasing the compactness, thus requiring a medium amount of space to account for the extra calculations. Despite still needing to convert the state, the use of the summation method for $\left\langle S_{z}\right\rangle$ still required less total space for calculation.

\section{Preferences in the spins context}

To analyze which computational features led to students' preferences for a given method, we focus on the 10 of the 20 students who used both methods when calculating $\left\langle S_{y}\right\rangle$ and $\left\langle S_{z}\right\rangle$ for a state written in the $y$ basis. Preference did not change between calculations of $\left\langle S_{y}\right\rangle$ or $\left\langle S_{z}\right\rangle$. When asked about preference in the interview, students had already been asked to perform both calculations $\left(\left\langle S_{y}\right\rangle\right.$ and $\left.\left\langle S_{z}\right\rangle\right)$ with both methods, and they were reflecting on their 
experiences. Five students preferred the summation method (although several of these students used the matrix method as their first method), two students preferred matrix, and three expressed no preference.

Students who preferred summation notation did so for its perceived ease and because it involved less mathematics where they could make mistakes.

Quincy: We can do this [sum] by any means. that's fantastic...It boiled down 8 lines to about 3... Also for me, it is much easier to make mistakes here [with matrices]. I think you corrected me twice...

Like Quincy, two other students who made smaller mathematical errors during calculation explicitly accounted for summation having a smaller room for mathematical error. Students' attention to the space and concern about mathematical error suggests preference here is tied to compactness and confidence, respectively.

Leanne and Tristan, who initially held more confidence in their matrix calculations due to increased familiarity, later switched their preference to summation because of relative speed of calculation.

Leanne: So like in terms of familiarity, like how much I've worked with different problems, this [matrix method] is much more familiar and because of that, it was my first instinct to go to but if I did approach this [summation] correctly this was much nicer to work with because it was much faster...

Leanne, and similarly Tristan, recognized the compactness of summation and upon accepting that the method gave them the correct answer, the compactness overrode the lack of computational confidence they felt at the beginning of the interview.

Only two students stated an explicit preference for matrix calculation over use of the summation method, despite this method being used the most often. Duncan expressed a lack of confidence with summation notation and later incorrectly used $\hbar / 2$ as the sole eigenvalue, preferred matrix notation as it was more "intuitive" than summation.

Duncan: It is just pretty intuitive once you get the matrix multiplication down because I can just imagine myself just carrying numbers like this and simplifies easier down to this and you end up getting the same answer. Even though there is a bit more work involved in the process, it is just something I can wrap my head around.

The intuition Duncan feels is a reference to the arithmetic procedures involved in multiplying matrices and not to the conceptual understanding as was the case with Mark in Sec. V B 3. To Duncan the lack of compactness of the matrix notation is inconsequential, since he still felt unsure of how to apply summation method, stating that most of his practice when solving for expectation value had been with matrix method. Notably, Duncan did not arrive at a correct answer with either method.
In contrast, Syd, who was proficient with both methods, preferred matrix notation for the simple matter that they felt it is always applicable.

Syd: I like the matrix one more because it works for any operator and vector, and this one [summation method] only works if you know everything: probability amplitude and all that stuff. $s$

In this case, Syd's preference is for a universal application, which is also mentioned by other students (e.g., Tristan while determining his preferences). As students typically see problems with states in the $z$ basis, the idea of universal application makes sense in that the matrix method would be less work for $\left\langle S_{x}\right\rangle$ and $\left\langle S_{y}\right\rangle$ since no conversions would be necessary. Beyond that, Syd's use of the matrix method for $\left\langle S_{y}\right\rangle$, where he wrote the $S_{y}$ operator in the $y$ basis, showed a level of comfort and fluency with matrices that only a few students showed in the interviews.

Three students did not express a strong preference for either matrix or summation notation and were able to acknowledge the pros and cons of each method.

Wayne: I would be more confident in this one [sum] simply because it saves less room for math or arithmetic mistakes, because here is a lot of multiplication and adding that I could have done wrong but here it [summation] is only two multiplications and only one addition, so it leaves less room for mistakes, but this one [matrix method] works always.

Wayne further stated that he would probably use both methods on an exam to be sure. Wayne's use of consistency checking is in line with past exam data where a small number of students used matrix method and then wrote "Check:" before verifying the calculation using summation method [12].

Mark and Owen also expressed no specific preference. While Mark felt that summation was more intuitive and Owen noted summation meant less room for mathematical errors, they both felt that they were comfortable with matrix notation and did not feel the additional calculation warranted a preference towards summation.

Overall, students' preference for a method was largely tied to either compactness or computational confidence. In several interviews, however, students' preferences and comfort shifted due to the externalization of a given method. In many cases, greater or equal comfort with matrix notation suggests that students' preferences are not simply a matter of compactness and efficiency, but that students often prefer methods that they feel are more familiar or practiced.

\section{RESULTS IN THE POSITION CONTEXT}

During the same semester as the interviews in the spins context, 12 more interviews were conducted as detailed in Sec. IV. All but two of the students belonged to the original interview pool. Students were first given a superposition of energy eigenstates $\left[\psi_{1}(x)=\frac{3}{5} \phi_{2}(x)-\frac{4}{5} \phi_{3}(x)\right]$, asked to 
solve for the expectation value of energy $\langle H\rangle$, and then subsequently the expectation value of position $\langle x\rangle$. All three methods can be used to calculate $\langle H\rangle$, but integration is the only viable method for $\langle x\rangle$. While the same expectation values were also asked for in the second portion of the interview with the parabolic wave function, $\psi_{2}(x)=$ $\sqrt{30 / L^{5}} x(L-x)$, because the parabolic wave function is purely a function of $x$, students' responses were most often restricted to the integration method. As such, we discuss students' implementation of the integration methods for $\psi_{1}$ and $\psi_{2}$ jointly.

Given the complexity that comes with integration and the time constraints of the interview, students were not asked to carry out the computation of any integrals. The focus of the interview instead was on a discussion of the different methods of calculating the expectation value. This means that computational compactness plays less of a role in our analysis of student work in the context of wave functions. That being said, integration would likely be labeled with a low compactness in any context, as is the wave function notation categorized by Gire and Price [1]. This is due to the amount of space it takes to multiply $\psi^{*}(x) \hat{A} \psi(x)$ before integration can begin. Conversely, for a superposition of two energy states, summation and an abbreviated matrix calculation would have a relatively high compactness. In the analysis that follows, we do not discuss compactness further and limit our discussion to identification, externalization, and computational confidence.

\section{A. Analysis of summation method for position}

The summation method was implemented by 11 of the 12 students and used almost exclusively for finding $\langle H\rangle$ for $\psi_{1}$ which was written in terms of energy eigenstates. This prevalence agrees with our previous findings [12]. Summation was the most efficient method in this case due to the ability to read the probability amplitudes of the specific eigenstates directly from the state. In the case of the parabolic wave function $\left(\psi_{2}\right)$, two students acknowledged the ability to write it in terms of energy eigenstates, but both stated summation is not a viable option given that the number of energy eigenstates is infinite.

Students' implementation of the summation method revealed similar challenges to the earlier spins context along the lines of identification and externalization, even among students who correctly used summation notation in the spins-context interviews earlier in the semester.

\section{Identification}

Students' identification for the summation method of $\langle H\rangle$ for $\psi_{1}$ was successful in all but two cases. Duncan, who incorrectly expressed eigenvalues in the spins context, incorrectly expressed the summation as $\Sigma c_{n} \phi_{n} \hat{H}$. He correctly identified the $\phi_{n}$ as the energy eigenfunctions and $c_{n}$ as the probability amplitude, but expressed he was not confident in this equation or summation method and did not continue with the calculation. Carlos incorrectly stated that the summation was of the coefficients and the energies, not recognizing that it was the probabilities that were needed. In the other cases where summation was invoked, the students were able to identify the needed quantities as part of the equation.

\section{Externalization}

As with the spins context, externalization accounts for representing the probabilities and eigenvalues for a given context. Since $\psi_{1}$ is given in terms of energy eigenstates, the probabilities for measuring the energies $E_{2}$ and $E_{3}$ correspond to the modulus square of the respective coefficients.

When solving for $\langle H\rangle$ for $\psi_{1}$, two students who had correctly used summation in the spins context, Leanne and Wayne, expressed the corresponding energy eigenstates in place of the eigenvalues (Fig. 5). Leanne justified that the functions (which were the eigenstates) could be included as part of the summation, drawing a parallel to integration in which she would "plug in a 0 or in this case an $L \ldots$ " and "the sine would go to a specific value." Wayne was not concerned with the presence of the functions until being presented with the correct answer later in the interview.

Victoria was the only student who struggled with determining the probabilities as part of the summation method for $\langle H\rangle$. Despite Victoria having correctly employed the summation method in the context of spins in a previous interview, rather than recognize that the coefficients are probability amplitudes, she tried to calculate the probability of each energy by drawing a graph for each energy eigenstate and solving for the area under each.

Feeling the most confident in using the summation method, Victoria attempted to use it to solve for $\langle x\rangle$ by first creating a bar graph by selecting 5 different positions along the wave function. She then talked through what the sum would mean in this context.

Victoria: Yes, so before let's say like 1, 2, 3, 4, 5. [Draws 5 vertical bars under the wave function]. If $i$ just had 1 and 5 , I would just do the probability of $1 \ldots$ times that eigenvalue plus probability of 5... (Fig. 6).

Here, Victoria correctly articulated what summation would look like for 5 discrete positions. She connected the heights of the bar graphs to the locations, but as she moved forward to add more bars, she lost track of how the individual eigenvalues become the function of " $x$." Instead, she then

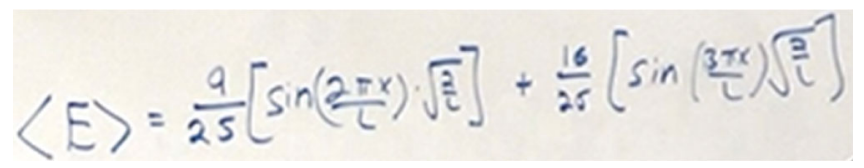

FIG. 5. Wayne's externalization of eigenstates in place of eigenvalues for the summation method. 


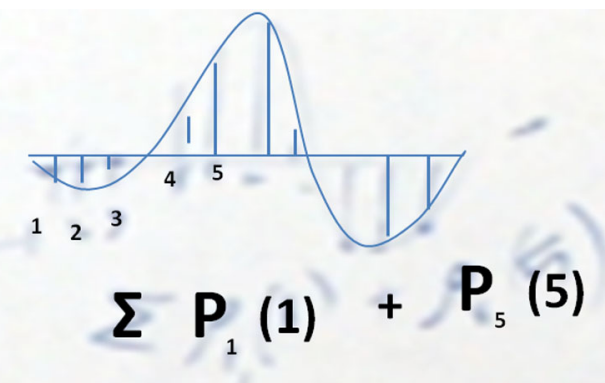

FIG. 6. Victoria created bars under the wave function to attempt summation for position. Typed numbers and symbols are added for legibility.

claimed the eigenvalue was the value of the wave function $\psi(x)$ and then could not resolve this with probability being $|\psi(x)|^{2}$.

While all other students stated summation for $\langle x\rangle$ was not possible, Tristan was able to conceptualize the summation over $x$ with slightly more success than Victoria, but did not attempt to express anything given that writing out the summation for position would require infinite terms.

Interviewer: Question for $\langle x\rangle$, could you use the summation method to think about that one?

Tristan: [Jokingly] You could if you had a really long board...that'd be like, technically you'd be adding every possible position that you could have but for these wave functions it would be like 0 to $L$, so you'd be adding infinitesimal positions.

While neither Tristan nor Victoria explicitly connected the expression for summation over $x$ to the integral, students' attempts here go a long way to show how using the understanding from discrete contexts can be used to leverage the meaning and structure of the integration method when calculating expectation value in a continuous basis.

Just as many students struggled to determine the probabilities and eigenvalues in the spins context, these issues persisted in the wave functions context. Furthermore, the added complexity of the continuous wave functions increases the ways in which students can calculate the probabilities, which can lead to additional confusion. Without an understanding of what the values and probabilities are in a given context, students cannot take advantage of the efficiency of the summation method for $\langle H\rangle$ and must resort to longer integral calculations.

\section{Computational confidence}

The majority of students responded that mathematics summation for $\langle H\rangle$ was relatively easy. Mark, having been interviewed previously in the context of spin, went further to state again that the summation method was still a very intuitive method of calculation. Conversely, the students who struggled with the application also expressed a lack of confidence. As with student responses in the context of spins, lack of confidence with summation was mostly due to a lack of familiarity or practice with applying the method.

Carlos: I don't use the sum method very often. It's just something that I happened to, it's just one of those things that I know is there but I'm not/ I don't feel comfortable enough with that way to do it

Carlos's discomfort, as well as that of Leanne's, follows naturally from their errors with externalization. They both expressed dissatisfaction when deciding what needed to be plugged in as the eigenvalues. Wayne, not aware of the error with his summation method or the inconsistency that his answer would not have been in units of the energy, did not share any discomfort and was still confident in his application of the summation method as the easier method.

\section{B. Analysis of the integral method for position}

Integration is universally applicable for any operator and state that can be expressed as continuous functions of a variable. This method was invoked by all students for both the expectation values of energy and position for both of the wave functions, except for one student who said $\langle H\rangle$ could not be found for $\psi_{2}$ since it was written as a function of $x$ and not energy.

\section{Identification}

The identification of the integration method was generally successful for all students. For integration, identification means distinguishing the terms of the equation as an integral of a product of $\psi^{*}(x) \hat{A}(x) \psi(x)$ over a certain variable, where $\hat{A}$ is an operator, and $\psi$ is a wave function. Identification does not require students to know the functions or the form of the operator, but only the setup of the equation. Almost all the students $(N=8)$ used the Dirac form as an explanation for their setup (Fig. 7). This aligns with findings from Gire and Price [1]. For students writing the integral from a Dirac template of $\langle\psi|A| \psi\rangle$, the notation provided structural support for students cueing the ordering of the integrals so that the operator was between the wave function and its complex conjugate.

Only two students, Victoria and Keith, struggled with identification. Victoria was unsure if the first instance of the wave function was supposed to be the complex conjugate

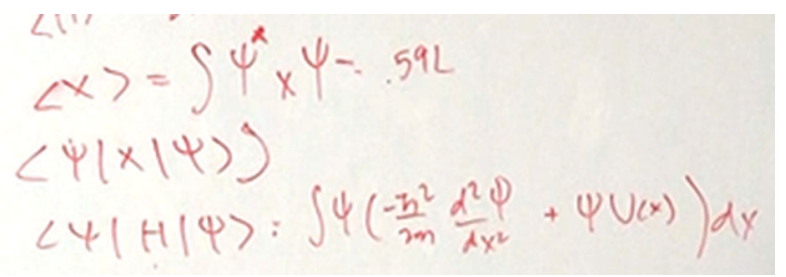

FIG. 7. Student work showing the use of Dirac notation when setting up integrals. 
and eventually decided the complex conjugate did not need to be included at all.

Keith neglected to include the actual integral for the integration method for $\langle H\rangle$ for the superposition state. Instead he left the method as the product of all of the terms and remained unsure of how to proceed with the calculation until later in the interview when he was calculating $\langle x\rangle$ for the same state. At first he wrote the same product, $\psi^{*} x \psi$, and then recognizing that $\langle x\rangle$ needed an integral, returned to $\langle H\rangle$ and added the integral. We label these as errors with identification since this aspect refers to identifying the correct structure of the equation.

\section{Externalization}

For the integral method, proper externalization accounts for inserting the correct wave function and its complex conjugate and for using the correct representation of the operator. In this context, this means using the representations as a function of position and integrating over $d x$. For $\hat{x}$ and $\hat{H}$ they are represented as $x$ and $-\hbar^{2} / 2 m$ $\partial^{2} / \partial x^{2}+V(\hat{x})$, respectively, in the position representation. Compared with both summation and matrix methods in the context of spins, once students had the form of the integral, only a few students had difficulty with externalization. Furthermore, all students correctly used $d x$ as the differential for both $\langle\hat{x}\rangle$ and $\langle\hat{H}\rangle$.

In the case of the superposition state $\psi_{1}$ all students used the correct sine functions and the correct $\hat{H}$ operator, both of which were written on an equation sheet provided to students. The increased success of externalization here, compared with work with the matrix method, is likely due to the students not needing to attend to basis for the integrals the way they needed to for the given spin state.

However, some students were unsure of how to represent the $\hat{x}$ operator in function notation. Piper, specifically, was not sure how to represent $\hat{x}$, since it was not provided on the equation sheet. Some students additionally were unsure of how to represent $\hat{H}$ before recognizing it was provided. It is likely that students would have had more difficulty with externalization if the $\hat{H}$ and the $n$th eigenfunction had not been provided, just as they may not have been able to recall the exact form of the $S_{y}$ matrix or representation of the $| \pm\rangle_{y}$ kets.

\section{Computational confidence}

Since students did not carry out any calculations, computational confidence was not directly assessed in the same manner as other methods. However, some students did note that they found integration straightforward or in some cases, as stated by Tristan, that the integration looked "really fun and interesting," indicating a level of comfort with this method.

Conversely, many other students expressed relief when being told they would not have to calculate any integrals or made comments regarding the amount of time it might take to compute the integral.

\section{Analysis of the matrix method for position}

When asked if matrices were an acceptable method of solution, most students responded that matrices were not applicable (even in the context of energy) due to the continuous nature of wave functions. A few students stated that the use of matrices was an acceptable method, but they would not know how to apply it in these situations. However, two students, Keith and Carlos, used matrix multiplication as a method of solution when calculating $\langle H\rangle$ for $\psi_{1}$.

Keith proficiently used matrices to calculate $\langle H\rangle$ for the superposition, showing growth in his application of matrices for solving for the expectation value when compared with his interview in the spins context. He first started with a four state system because the state did not have any energy eigenstates higher than four. Upon recognizing that the corresponding coefficients for $E_{1}$ and $E_{4}$ were also 0 , he transitioned to a two state system for the purpose of calculation.

Carlos initially struggled with the externalization of the matrix for the Hamiltonian matrix. He first represented $\hat{H}$ as the partial derivative that was used for integration, but was unable to proceed. After targeted questions about what quantities the Hamiltonian measures, he was able to arrive at the correct matrix using the energy eigenvalues and carry out the calculation.

Both Keith and Carlos expressed comfort with matrix notation. For Carlos, who was not interviewed in the first round, he expressed that this comfort came from his experiences with matrices in the context of spins.

Carlos: Well with the spin it is a lot easier for me to remember because with the spin everything is still very easily representable in matrix notation, because with the spin when you have this one it comes out to. I just rewrite it. I just jump straight into matrix notation and the math just flows for me.

Despite not being able to represent the matrix at first, Carlos expressed that using the matrices in this context were helpful. Matrices here led Carlos to fix his identification error with the summation method and find some confidence in the method. The matrix method also provided Keith with an alternative method to integration since he was not able to implement the summation method for $\psi_{1}$. Both students were able to correctly implement the matrix method as compared with summation, which further supports students have increased confidence in matrix notation.

\section{Preferences in the position context}

To address students' preferences, we focus exclusively on the calculation of the expectation value of energy for the state written as a superposition of energy eigenstates, $\psi_{1}$, 
since multiple methods are possible. Eleven of the twelve students were able to invoke more than one method. One student (Carlos) mentioned all three methods and made a reasonable attempt at each. Five students shared an explicit preference for summation over the other methods.

As was the case for the spins context, students' stated preferences for summation were often tied to the ease or speed of the calculation and compactness. Carlos's preferences changed throughout the interview and eventually settled on summation for its mathematical simplicity. At first, he was uncomfortable with summation, but later was able to fix his mistake when comparing his use of the matrix method to the elements of the summation. At this point, his preference switched to summation, despite an initial greater confidence with matrices.

Interviewer: Now that we have the summation right too, is there a particular one that you have a preference for?

Carlos: Summation.

Interviewer: Okay, why?

Carlos: It's way faster, if I had done it right.

Four other students shared similar preferences for summation. For example, Mark's preference was tied inherently to the perceived intuitive ease of summation and Piper preferred summation because she was able to recognize the "jumping off points, so we don't have to do the whole integral thing," referring to the ability to read out the probability amplitudes directly from the state.

Keith, having not invoked summation, preferred the matrix method over integration for the same reason as the other students preferring summation: "It just seems a lot easier than dealing with integrals."

Three students shared a preference for integration over the summation method. Duncan felt the integral was "more straightforward... even though doing all the integration is kind of a pain" and Leanne stated the integration method made it "easier for me to keep track of what I need to be doing." These two students had trouble with the summation method either by not being able to identify the right equation or in attempting to express the eigenvalues. Students, if aware of their difficulties with a particular method, preferred the one in which they were able to correctly identify and externalize. This led both Leanne and Duncan to a preference for integration since they were more confident in being able to express the terms correctly.

Freya's preference for integration was based solely on having learned integration first when finding expectation values for wave functions.

Freya: I think I always just do integral even though I think the probability value one sums to 0 , but for some reason I just go to the integral... I think it was actually the order that I was taught in, because I started, I learned how to do the integral first and then the probability later. And so in my head that one just comes first.
While Freya's preferences may be based on confidence, her discussion also suggests more of an emphasis on a "plugand-chug" mindset where the emphasis is on inserting the wave functions into the integral and carrying out the calculation rather than using reasoning to carry out a shorter calculation. This is analogous to matrices in the spins context where the method appears to work in all cases.

Two students did not have an explicit preference for either summation or integration and highlighted aspects of each method that they liked. Tristan admitted that summation looked "more practical" in that there would be less math but stated that he would probably choose the integration because it looked "really fun and interesting."

Just as was the case with matrices, some students prefer integration due to increased familiarity and confidence over the efficiency of summation method. In some cases, like with Freya, this is simply due to its repeated use in other portions of the course where it is used for normalization and calculating probabilities. Tristan's preference for integration indicates an increased comfort that overrides the extra space needed for calculation. However, in a majority of cases where students are equally proficient with summation and with integration, desire for compactness led to a preference for summation. Conversely, some students turn away from the summation method due to difficulty with the externalization process. This struggle with the externalization is evidenced by errors expressing the eigenvalues and probabilities for energy eigenstates just as with the eigenstates of the spin operators.

\section{DISCUSSIONS AND CONCLUSIONS}

Application of the computational features framework provided detailed insight into students' calculation of expectation values and allowed us to identify the aspects of calculation which contribute to student preferences for different methods. The framework was designed to characterize students' implementation of methods across the dimensions of identification, externalization, computational confidence, and compactness. While identification and externalization allow for greater analysis of students' understanding of a given method, confidence and compactness were the features that largely drove student preference.

As part of the adapted framework, identification and externalization attend to students' modeling of the physical system into the form of an equation for computation. Identification allows us to account for whether students understand the equation and its components, while externalization accounts for representing those components in terms of a given context (e.g., basis of a vector or measurable values for a given operator). The framework further includes compactness and computational confidence as a means of addressing the amount of space it takes to carry out a computation and whether students are confident using the associated mathematics, respectively. The latter elements were designed to assess aspects that would influence 
students' preferences for a given method and would correspond to the reasons why students "activate a given tool" from the ACER framework [13], where students chose a given equation. We do not claim that these are the only four elements that describe students computational work, but we found these sufficient for characterizing students preferences. This work has shown how the computation features can be used to describe implementation of a given method of solution and be used to explore student choices when there are multiple methods that could be used.

Analysis of 32 interviews conducted over two points during a quantum mechanics course offer some answers. Our research questions are as follows:

1. What possible challenges do students face when implementing different methods?

2. What aspects of a given method influence students' preferences?

3. If students use a method that is not their preferred method, what influences their choice?

To answer the first question, we found the largest challenge for most students was with externalization, (e.g., representing vectors in the right basis or expressing the correct eigenvalues), or with identifying the right components in each expression. This was true for all three methods (matrix, summation, and integration) and was the main hindrance to correct implementation. Answers to research questions (2) and (3) are nuanced and intertwined. We synthesize some aspects of these answers in the following paragraphs.

Largely, students' preferences were guided by either compactness or confidence. In relatively few cases, students possessed a strong understanding of multiple methods and shared that they did not have a preference but stated that depending on the context, different methods would be valuable for different reasons. We find this line of reasoning to be closest to an expert perspective, since these students were able to acknowledge the benefits and drawbacks of each method. The role of compactness in an expertlike response was in recognizing that the summation method would take up the least amount of space and computational effort.

Despite several students arriving at the conclusion that summation would have been better in some instances, many had still started with a matrix calculation due to an increased familiarity with the method and confidence with the matrix calculation. In cases where students have a strong understanding of both matrix and summation methods, such as Syd or Wayne, there was an inclination towards the matrix method after stating that it is applicable in any context. The draw of the matrix method for these students was that it was a method they could learn that would work in any context that was given. This draw is inline with problems and examples students see as part of the course where they typically work with states that are already represented in the $z$ basis. When this is the case, the matrix method is indeed more efficient for expectation values for measurements along different axes, since $\left\langle S_{y}\right\rangle$ and $\left\langle S_{x}\right\rangle$ are commonly given in the $z$ basis, and students can forgo converting the state or doing the added work of finding probabilities.

More commonly, student preferences for matrix notation were related to an increased amount of practice or general familiarity with matrix notation. This preference is to use a "tried and true" method of calculation rather than a new, untested method. By the time the expectation value is introduced in a spins-first curriculum, students have been consistently using both matrix and Dirac notation to carry out the majority of calculations. At this point in a typical spins-first course, the summation method is now the novel concept. Even students who were able to correctly describe and apply the summation method first applied the matrix method when given the problem. It was only when they were specifically asked about additional methods that they invoked summation. This suggests that some students may not initially think of the summation method when approaching an expectation value problem simply because they have not used the summation method as frequently. This kind of preference appears again for some students in the wave function context, where they find the integration method to feel more familiar from other calculations.

Associated with the idea of familiarity, a third reason for an increased preference for matrix notation may be based on a cueing by the notation used. Familiarity was additionally responsible for students' use of the matrix method first, even when they later used and shared preference for the summation method. The notational representation for the expectation value of an operator $\hat{A}$ for a state $\psi$ is written as $\langle\hat{A}\rangle$. This notation is an abbreviated form of $\langle\psi|\hat{A}| \psi\rangle$. The symbols $\langle$ and $\rangle$ are direct references to the bra and ket used in the expectation value. As stated above, students have become familiar with the use of matrix notation to carry out operations in Dirac notation. Thus, even just the representation of $\langle\hat{A}\rangle$ could signal the use of matrices to students less familiar with other methods. Three students initially were unable to connect the question of expectation value to its mathematical representation, but when they were explicitly prompted with the notation " $\left.\left\langle S_{y}\right\rangle\right\rangle$ " all three invoked the matrix method after the expansion to the bra-ket representation $\langle\psi|\hat{A}| \psi\rangle$. None of these three students invoked summation notion when asked about the expectation value. We hypothesize this is because there is nothing inherent about the notation that connects to a summation method, despite the work in Dirac notation to arrive at Eq. (2). Given the prior experience switching between Dirac and matrix notation throughout the course, plugging in matrices becomes a matter of pattern recognition and is reduced to a simple arithmetic process.

Additionally, the matrix method likely has an increased prevalence among student responses specifically because it involves a limited amount of conceptual work and can be 
used as a purely mathematical procedure. Interviews revealed that many of the students did not attend to the basis representation of the vectors or matrices when carrying out matrix multiplication and that other students struggled or failed to identify eigenvalues which were already embedded in the matrices. Checking for matched bases between matrices and vectors is often unnecessary when working with an initial state in the $z$ basis. Students were less likely to be aware of their errors with externalization when applying the matrix method than when using summation, which led to a number of students expressing a false confidence in matrix notation. Externalization for matrices requires attending to basis, but even without doing so, they can still carry out the matrix calculation and arrive at an answer. A student applying summation cannot proceed with the calculation if they cannot express the requisite eigenvalues that can be measured for a system.

Errors with externalization are much more of an impediment while working with summation since they are fundamental to the calculation. Both Piper and Raife were able to correctly work through the matrix notation and arrive at an answer including $\hbar / 2$ but were unable to see the role of $\pm \hbar / 2$ in the summation method. Piper was unsure what the eigenvalues were for the state, while Raife was unsure of their place in the summation calculation. Other students, like Duncan, shared a preference for matrix notation when they expressed a lack of confidence or displayed difficulty with expressing components of the summation method. This suggests an affordance of the matrix method for students is the ability to carry out the calculation without attending to the meaning of the elements. In essence the meaning of the eigenvalues and probabilities can be overlooked since matrix calculation is not impeded by a lack of being able to identify these components.

Barring those who had difficulties with eigenvalues or probabilities, students more often preferred the summation method due to the expediency of the calculation in specific contexts. However, it was not invoked by all students, showing that students do not necessarily recognize it as an applicable solution to solving for the expectation value. Further, even though students indicated that they preferred summation, most of these students initially used the matrix method and only invoked summation when asked for an additional method. The use of matrix notation first, despite preference for summation, suggests that preference is not the only important factor in selecting a method of calculation. Because of the prevalence of matrices in spins-first quantum mechanics instruction, it is possible that many students will still default to the matrix method. This hypothesis could be tested by interviewing students from a position-first instructional paradigm.

\section{IMPLICATION FOR INSTRUCTION}

Our results show that some students do not take advantage of the conceptual meaning of the expectation value or connect it to the mathematics used for calculation. Students' lack of confidence in the identification of eigenvalues and their associated probabilities lead them to select methods that allow them to plug and chug from the information given in the question. Increased instructional emphasis on the meaning of the expectation value may lead to an increase in students recognition and preference for the summation method in situations where it is more efficient. Students' struggles with the summation method is most often tied to a lack of confidence with or misidentification of the eigenvalues. Increased emphasis on what the eigenvalues are in different contexts could lead to an increase in students' recognition and preference for the summation method is situations where it is more efficient. Other students appeared to have difficulty with the summation method when it was given in its compact symbolic notation or do not have a deep connection with the "weighted average" concept of expectation value when they feel it is a novel approach.

The errors that occurred when using the matrix method were almost exclusively tied to inconsistent basis use. Gire and Price identified that matrices have very low externalization in their framework as the basis for vectors and matrices is hidden. In a spins-first quantum mechanics course, very often matrices are only expressed in the $z$ basis, and students may not recognize that this is an arbitrary choice, and that the basis choice is something they need to attend to when solving problems. Instructors should be cognizant of this tendency and incorporate exercises and examples that use matrices in different bases.

In other cases, tying summation, matrix, and integral methods together by explicitly showing how the mathematics of matrices leads to summation and integration would be useful for students in understanding the meaning of each method in different contexts. While previous literature has shown students' incorrectly ordered the elements as part of integration [8], our interviews showed that many students who were able to connect the representation of expectation value in terms of the Dirac notation used in the spins context were able to consistently write their integrals correctly. Generally, students attempting to apply summation or matrices in continuous cases were able to tie their conceptual understanding of discrete and continuous bases more closely together. This was specifically the case when students, like Victoria or Tristan, tried to connect bar graphs to wave functions. Using students' familiarity with matrix notation seems a promising bridge to the mathematics of the continuous position space and could lead to increased comfort and intuition with other methods of computing expectation values. We plan to develop such activities to structure and support students' understanding of the expectation value and in terms of both conceptual meaning and mathematical representation. 


\section{ACKNOWLEDGMENTS}

This material is based upon work supported by the National Science Foundation Grants No. DUE 1626280, No. 1626594, and No. 1626482.

[1] E. Gire and E. Price, Structural features of algebraic quantum notations, Phys. Rev. ST Phys. Educ. Res. 11, 020109 (2015).

[2] C. Singh, Student understanding of quantum mechanics at the beginning of graduate instruction, Am. J. Phys. 76, 277 (2008).

[3] C. Singh, Student understanding of quantum mechanics, Am. J. Phys. 69, 885 (2001).

[4] G. Zhu and C. Singh, Improving students' understanding of quantum measurement. I. Investigation of difficulties, Phys. Rev. ST Phys. Educ. Res. 8, 010117 (2012).

[5] C. Singh and E. Marshman, Review of student difficulties in upper-level quantum mechanics, Phys. Rev. ST Phys. Educ. Res. 11, 020117 (2015).

[6] C. Singh, Student difficulties with quantum mechanics formalism, AIP Conf. Proc. 883, 185 (2007).

[7] E. Marshman and C. Singh, Investigating and improving student understanding of quantum mechanical observables and their corresponding operators in Dirac notation, Eur. J. Phys. 39, 015707 (2017).

[8] H. Sadaghiani, G. Passante, and S. Pollock, Student understanding of quantum mechanical expectation values, in Proceedings of the 2018 Physics Education Research Conference, Washington, DC, edited by A. Traxler, Y. Cao, and S. Wolf (AIP, New York, 2018), https://doi.org/ 10.1119/perc.2018.pr.Sadaghiani.
[9] H. Sadaghiani and L. Bao, Student difficulties in understanding probability in quantum mechanics, AIP Conf. Proc. 818, 61 (2006).

[10] E. Marshman and C. Singh, Investigating and improving student understanding of the probability distributions for measuring physical observables in quantum mechanics, Eur. J. Phys. 38, 025705 (2017).

[11] T. Wan, P. J. Emigh, and P. S. Shaffer, Investigating how students relate inner products and quantum probabilities, Phys. Rev. Phys. Educ. Res. 15, 010117 (2019).

[12] G. Passante, H. Sadaghiani, S. Pollock, and B.P. Schermerhorn, Students' choices when solving expectation value problems, in Proceedings of the 2018 Physics Education Research Conference, Washington, DC, edited by A. Traxler, Y. Cao, and S. Wolf (AIP, New York, 2018), https://doi.org/10.1119/perc.2018.pr.Passante.

[13] B. Wilcox, M. Caballero, D. Rehn, and S. Pollock, Analytic framework for students' use of mathematics in upper-division physics, Phys. Rev. ST Phys. Educ. Res. 9, 020119 (2013).

[14] E. F. Redish and E. Kuo, Language of physics, language of math: Disciplinary culture and dynamic epistemology, Sci. Educ. 24, 561 (2015).

[15] O. Uhden, R. Karam, M. Pietrocola, and G. Pospiech, Modelling mathematical reasoning in physics education, Sci. Educ. 21, 485 (2012).

[16] D. H. McIntyre, Quantum Mechanics (Pearson Education, Inc., San Francisco, CA, 2012). 